\title{
FOCUSING SOLENOIDS FOR THE MICE COOLING CHANNEL
}

 \\ ${ }^{1}$ Lawrence Berkeley National Laboratory, Berkeley CA 94720, USA \\ ${ }^{2}$ Rutherford Appleton Laboratory, Chilton-Ditcot, OX11 0QX, UK \\ ${ }^{3}$ Oxford University, Oxford, OX12JD, UK
}

\begin{abstract}
This report describes a design for focusing solenoids for the low beta sections for the proposed Muon Ionization Cooling Experiment (MICE). There are three focusing solenoid pairs that will be around the muon absorbers for MICE. The two solenoid coils have an inside diameter of $510 \mathrm{~mm}$, a length of $180 \mathrm{~mm}$, and a thickness of $100 \mathrm{~mm}$. A distance of $260 \mathrm{~mm}$ separates the two coils in the pair. The coils are designed to operate at opposite polarity, in order to create a gradient field in the low beta sections of the MICE cooling channel. As result, the force pushing the coil pair apart approaches 270 metric tons when the coils operate close to the short sample current for the superconductor. The forces between the coils will be carried by a support structure that is both on the inside and the outside the coils. During some modes of operation for MICE, the coils may operate at the same polarity, which means that the force between the coils pushes them together. The focusing magnet must be designed for both modes of operation. This support structure for the coils will be part of the focusing magnet quench protection system.
\end{abstract}

\section{INTRODUCTION}

The Muon Ionization Cooling Experiment (MICE) is a channel of superconducting solenoid magnets. The magnets in MICE are around the RF cavities, absorbers (liquid or solid) and the primary particle detectors [1], [2]. The MICE superconducting solenoid system consists of eighteen coils that are grouped in three types of magnet assemblies. The cooling channel consists of two complete cells of an SFOFO cooling channel. Each cell consists of a focusing coil pair around an absorber and a coupling coil around a RF cavity that reaccelerates the muons to their original momentum. At the ends of the experiment are uniform field solenoids for the particle detectors and a set of matching coils used to match the muon beam to the cooling cells. Three absorbers are used instead of two in order to shield the detectors from dark currents generated by the RF cavities at high operating acceleration gradients. A layout of the full version of MICE is shown in FIG 1

The MICE superconducting solenoids will be fabricated in modular units. There are three reasons for wanting the solenoids in modular units. First, three different groups in Europe and the United States will fabricate the solenoid magnets. Second, there will be several operating steps for MICE. Third, the absorbers, which are housed within the focusing modules, must be changed from liquid absorbers to solid absorbers. This requires that the focusing magnet modules be removable from the MICE. 


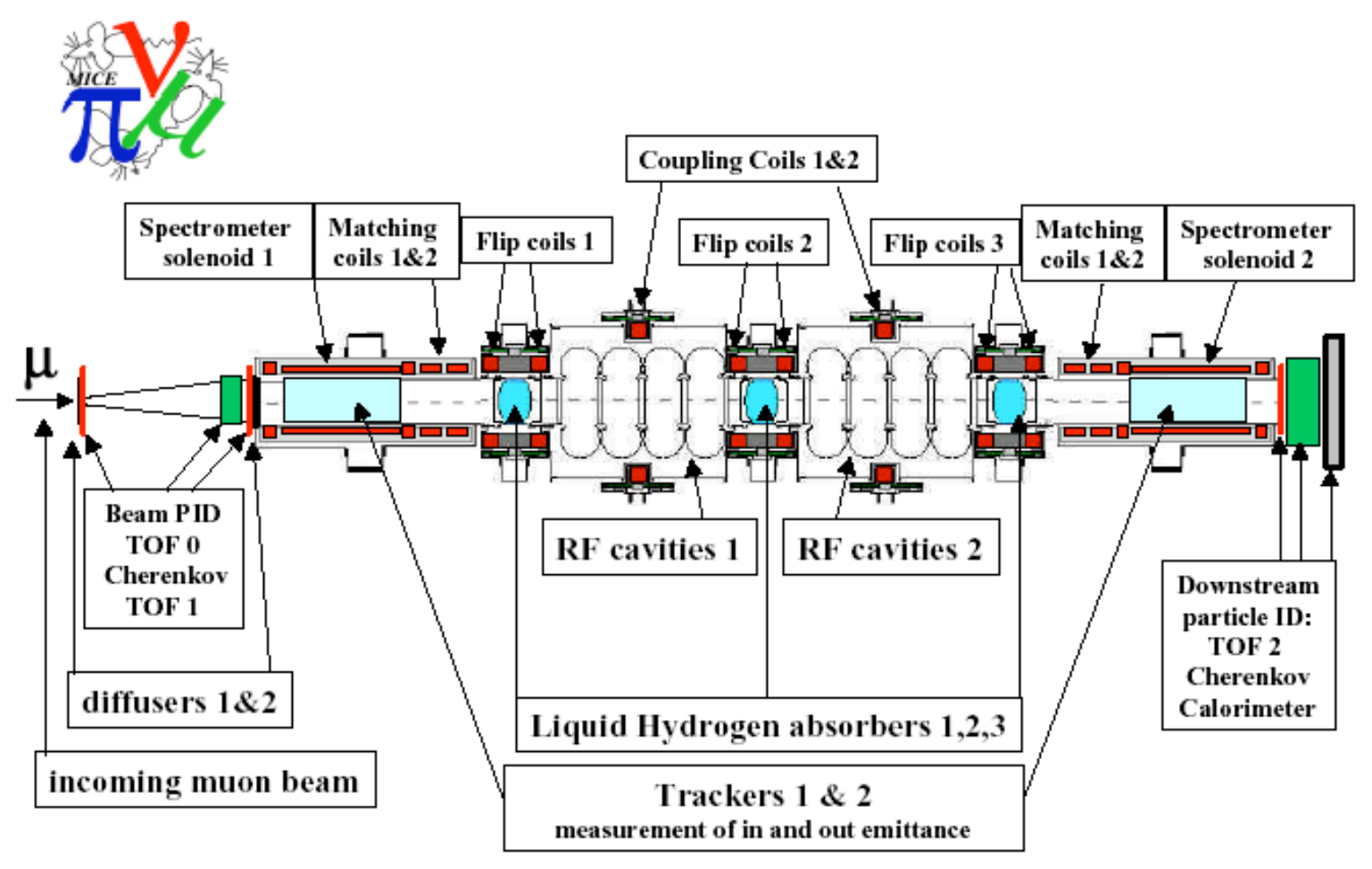

FIGURE 1. A general layout of the full MICE experiment showing all eighteen superconducting solenoid coils in seven magnet modules, the conventional RF cavities, the liquid hydrogen absorbers, the two central trackers which are in a uniform magnetic field and other types of particle detectors

The focusing solenoid modules are shown in FIG 1 as Flip Coils 1, Flip Coils 2, and Flip Coils 3. The magnet coils are around Liquid Hydrogen Absorbers 1, 2, and 3. The reason that the focusing coils are described as flip coil is that during normal operation of MICE the magnetic field flips from one polarity to the others as one travels down the bore of the magnet. The magnetic field is zero at the magnetic center of the focusing solenoid pair. The field flip produces a focused muon beam region that has a relatively low beta. (The muon beam beta will vary from $420 \mathrm{~mm}$ to as low as $55 \mathrm{~mm}$ during the life of the experiment.) There are times during the life of the experiment that the coils in the focusing coil pair will be operating at the same polarity. This implies that the solenoid pair should be designed to operate over a range of currents and at various coil polarities.

The absorbers that are within the bore of the focusing solenoid pair may be either liquid absorbers (hydrogen or helium) or solid absorbers (lithium, lithium hydride, beryllium, or plastic) [3]. The liquid absorbers are discussed further in references [4] and [5]. The design of the solenoid pair is determined by the need for a liquid hydrogen absorber within the magnet bore and the allowable space between the RF cavity modules (see Figure 1)

The liquid hydrogen absorbers must be located within the 470-mm diameter warm bore of the focusing solenoid. The focusing solenoid module is designed so that the Liquid hydrogen or solid absorbers can be installed as a fully assembled device from one end of the magnet. The cryogenic piping and the gas neck for the absorber goes past the end of the magnet vacuum at one end of the focusing module. In order to do this, the solenoid is not centered in the module vacuum vessel. The magnet center is shifted $32 \mathrm{~mm}$ along the axis from the center of the module. The absorber (liquid or solid) is located in the vacuum space between the two RF cavities or between one RF cavity and a detector module.

FIG 2 shows a cross-section of the focusing module. The cross-section includes the axis of rotation for the system and is taken approximately 45 degrees from the vertical through the cold mass supports. The liquid hydrogen absorber is shown installed in the magnet. The physical length of the coil package is about $690 \mathrm{~mm}$. The end to end length of the magnet cryostat vacuum vessel is about $720 \mathrm{~mm}$. This leaves about $62 \mathrm{~mm}$ of space at one end of the module for the absorber plumbing to go past the end of the magnet cryostat vacuum vessel. 


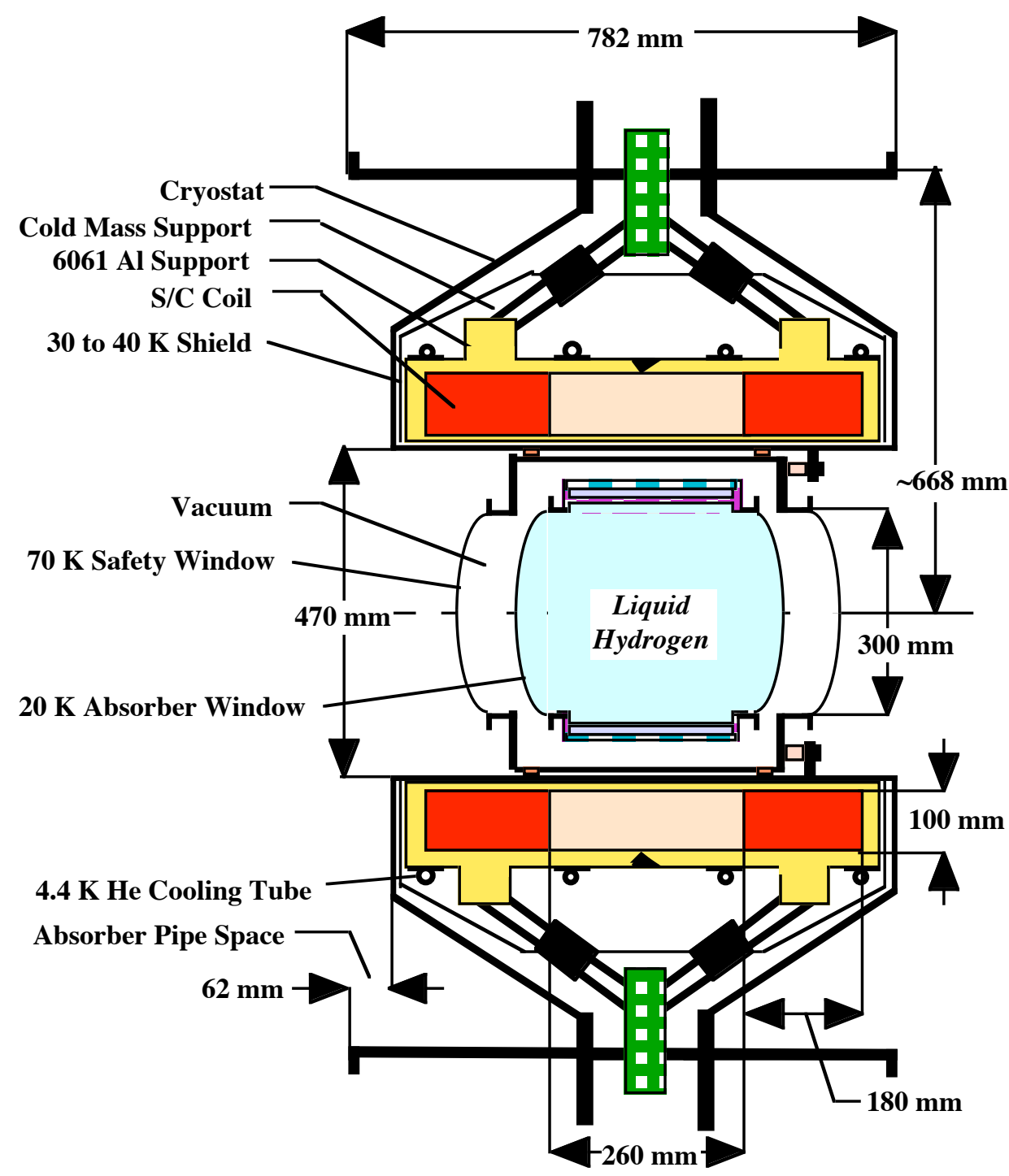

FIGURE 2. A cross-section of the MICE focusing module showing the S/C coils and a liquid absorber

\section{A COMPARISON OF THE FOCUSING MAGNET TO THE LAB G MAGNET}

The MICE focusing solenoid shown in FIG 2 closely resembles a solenoid built for LBNL for a high gradient RF cavity experiment in Lab G at Fermilab [6] [7]. A cross-section of this magnet and its cryostat is shown in FIG 3. The Lab G solenoid produces a gradient field that is similar to the field that is required for MICE (particularly the case where the average muon momentum is $200 \mathrm{MeV} / \mathrm{c}$. As with the magnet that is shown in FIGURE 2 the Lab G magnet produces gradient field is generated when the two coils are operated at opposite polarity. Like the Lab G magnet, the MICE focusing may be required to operate as a two coil solenoid with both coils operating at the same polarity.

The biggest difference between the two systems is the design of the cryostat. The Lab G solenoid cryostat is $1038 \mathrm{~mm}$ long versus a proposed length for the MICE focus coil cryostat length of $720 \mathrm{~mm}$. Both magnets use a self-centering tension strap cold mass support system. The difference in cryostat length for the two systems is dictated by the fact that the Lab G coil supports are attached to the ends of the coil package. The MICE focus solenoid cold mass supports will be attached to the outer support cylinder for the coils. Because the Lab G solenoid cryostat length is not an issue, more space was also allocated for the intermediate temperature shields and multi-layer insulation in the Lab G solenoid. 


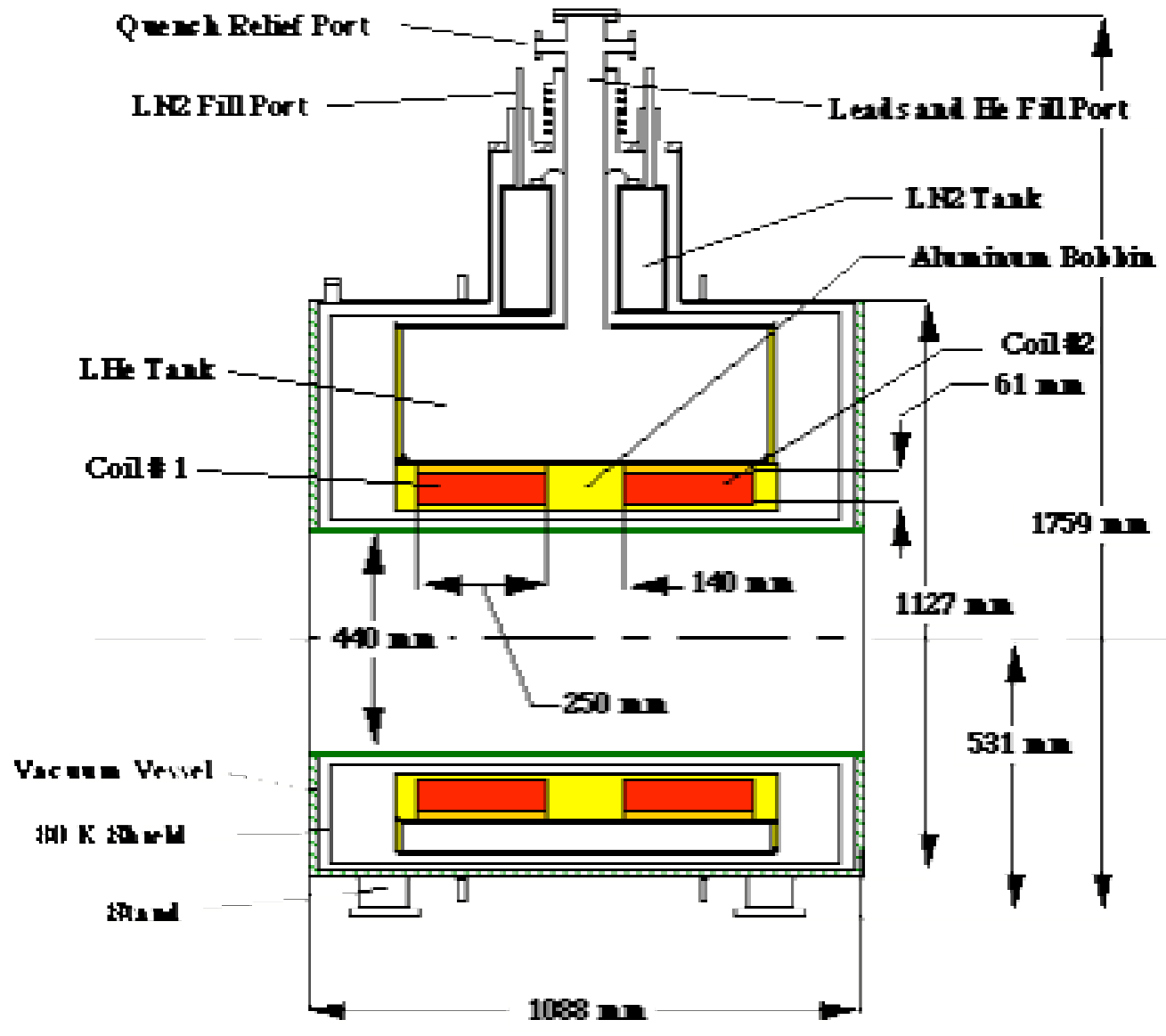

FIGURE 3. A cross-section of the gradient solenoid built for the $800 \mathrm{MHz}$ RF experiment in Lab G

The MICE focus solenoid warm bore is $470 \mathrm{~mm}$ versus $440 \mathrm{~mm}$ for the Lab G solenoid. The bobbin thickness for the Lab G magnet is $15 \mathrm{~mm}$ and the space for the cryostat wall and the insulation is $25 \mathrm{~mm}$. Less space is proposed for insulation and the warm bore tube for the MICE (about $15 \mathrm{~mm}$ ) and the aluminum bobbin inside the coil is only $5 \mathrm{~mm}$ thick. The reason for this is space between the outside of the hydrogen absorber and the inside of the coil must be minimized. The reason for the thinner coil bobbin in MICE is that it is proposed that the focus coils be wound and potted then they are installed into an outer support structure. The 5-mm thick inner aluminum carries a portion of the inter-coil longitudinal force thus reducing the thickness of the coil end plate. If the MICE coil were to be wound on the bobbin, the bobbin end plates could be a few millimeters thinner.

\section{TWO APPROACHES FOR BUILDING THE MICE FOCUSING COILS}

The differences between the Lab G coil and the proposed MICE focusing coils reflect in part differences in the design philosophy for the two solenoids. The differences between the Lab G coil design and the proposed MICE focusing coil design are as follows: 1) The MICE focusing solenoid coil bore is designed to be $510 \mathrm{~mm}$ versus $520 \mathrm{~mm}$ for the Lab G solenoid. 2) The length of the coil package proposed for the MICE focus coil package is $620 \mathrm{~mm}$ versus $640 \mathrm{~mm}$ for the Lab G solenoid. 3) The coils in the Lab G solenoid are 250-mm long versus a coil length of $180 \mathrm{~mm}$ for the MICE solenoid. 4) The current center for the Lab G solenoid is at a radius of $291 \mathrm{~mm}$ versus an average coil radius $305 \mathrm{~mm}$ for the MICE focusing magnet. 5) The biggest difference between the two coils is the space between the two coils. The lab G solenoid coils are separated by $140 \mathrm{~mm}$; the proposed spacing for the MICE coils is $260 \mathrm{~mm}$. 6) The MICE coils are $100 \mathrm{~mm}$ thick versus $62 \mathrm{~mm}$ for the Lab G solenoid coils. This difference is in part reflected by the fact that the Lab G coils are $250 \mathrm{~mm}$ 
long versus $180 \mathrm{~mm}$ for the MICE coils. In terms of overall coil design, the two magnet coil systems are similar, but the proposed MICE focusing coils will have fewer ampere-turns.

A layer of 5456 aluminum banding wound on the outside of the coil helps to control the magnet hoop strain. It is proposed that the MICE coil hoop forces be transmitted directly to the 6061 support structure on the outside of the coil. The 300 metric ton longitudinal force (pushing the coils apart) in the Lab G magnet is carried by the $15 \mathrm{~mm}$ thick bobbin and the 10 $\mathrm{mm}$ cylinder of 6061 aluminum that is on the outside of the coil. The design longitudinal force $(270$ metric tons at a muon momentum of $240 \mathrm{MeV} / \mathrm{c})$ between the MICE coils will be carried by the 25 -mm thick outer support structure and the inner structure that is a $5-\mathrm{mm}$ thick aluminum cylinder on the inside of the coils.

The conductor for the Lab G solenoid was a standard niobium titanium MRI conductor produced by IGC (now Outokumpu). This conductor has the following characteristics: 1) The bare dimensions of the conductor are $0.955 \mathrm{~mm}$ by $1.60 \mathrm{~mm}$. The conductor has rounded ends and is insulated with a Formvar resin that is $0.025 \mathrm{~mm}$ thick. 2) The ratio copper to superconductor is 4.0. 3) The residual resistance ratio (RRR) of the copper matrix is greater than 100.4) The number of filaments in the matrix is 55. The filament diameter is $87 \mu \mathrm{m}$. 5) The conductor twist pitch is $1.27 \mathrm{~mm}$. 6) The minimum guaranteed conductor critical current at $5 \mathrm{~T}$ and $4.2 \mathrm{~K}$ is $760 \mathrm{~A}$. (The $\mathrm{J}_{\mathrm{C}}(5 \mathrm{~T}, 4.2 \mathrm{~K})>2850 \mathrm{~A} \mathrm{~mm}^{-2}$ ) It is proposed that the same conductor as the Lab G magnet be used in the MICE focus coils, because this conductor is relatively inexpensive and available.

TABLE 1 compares the parameters for the Lab G solenoid operating at its design current of 265.0 A with the MICE focusing solenoid operating at its design current (the case where the average muon momentum is $200 \mathrm{MeV} / \mathrm{c}$ ) of $240.3 \mathrm{~A}$. Figure 4 show the conductor load lines for the two magnets operating in the gradient mode.

TABLE 1. A Comparison of the parameters for the MICE focusing magnet and the Lab G magnet

\begin{tabular}{lcc}
\multicolumn{1}{c}{ Magnet Parameter } & Focusing Magnet & Lab G Magnet \\
Inner Cryostat Radius (mm) & 235 & 220 \\
Outer Cryostat Radius (mm) & 668 & 564 \\
Cryostat Length (mm) & 720 & 1088 \\
Inner Cold Radius of the Coils (mm) & 255 & 260 \\
Average Coil Radius & 305 & 290.5 \\
Superconducting Coil Length (mm) & 180 & 250 \\
Superconducting Coil Thickness (mm) & 100 & 61 \\
Number of Layers per Coil & 72 & 58 \\
Number of Turns per Coil & 109 & 147 \\
Design Magnet Coil in Gradient Mode $\mathrm{I}_{\mathrm{d}}(\mathrm{A})$ & 240.3 & 265.0 \\
Peak Induction in the Coil at $\mathrm{I}_{\mathrm{d}}(\mathrm{T})$ & 6.27 & 6.52 \\
Overall Coil Current Density at $\mathrm{I}_{\mathrm{d}}\left(\mathrm{A} \mathrm{mm} \mathrm{mm}^{-2}\right)$ & 106.7 & 148.2 \\
S/C Matrix Current Density at $\mathrm{I}_{\mathrm{d}}\left(\mathrm{A} \mathrm{mm}^{-2}\right)$ & $\sim 180$ & $\sim 198$ \\
Magnet Self Inductance $(\mathrm{H})$ & $\sim 52$ & 68.5 \\
Stored Energy of the Magnet at $\mathrm{I}_{\mathrm{d}}(\mathrm{MJ})$ & $\sim 1.5$ & 2.40 \\
Design Temperature Margin at $\mathrm{I}_{\mathrm{d}}(\mathrm{K})$ & $\sim 1.1$ & 1.3 \\
Force Pushing the Coils Apart at $\mathrm{I}_{\mathrm{d}}(\mathrm{MN})$ & 1.82 & 3.00 \\
Cold Mass Support System Design Force $(\mathrm{MN})$ & $>0.60$ & $>0.25$
\end{tabular}

The total design ampere turns per coil for the Lab G solenoid is about 2.23 MA, whereas the design (at an average muon momentum of $200 \mathrm{MeV} / \mathrm{c}$ ) ampere turns per coil for the focusing solenoid shown in Figure 2 is $1.89 \mathrm{MA}$. The average current density in the two coils is quite different $107 \mathrm{~A} \mathrm{~mm}^{-2}$ for the proposed MICE focus coils versus $148 \mathrm{~A} \mathrm{~mm}^{-2}$ for the Lab G coils. The primary difference is the thickness of the glass between the coil layers. The Lab G coils were made as wet lay up, with the filled epoxy put between each layer. The design for the MICE focusing coils is based on vacuum impregnation of the coils after winding, so it is proposed that a thicker layer of glass cloth between the layers to carry the resin into the winding during vacuum impregnation. If the proposed MICE coils are wound like the Lab G coils, the coil thickness would be about $76 \mathrm{~mm}$ instead of $100 \mathrm{~mm}$. 




FIGURE 4. The Load Line for the Focusing Magnet and the Lab G Solenoid as Built (Both coils use the same conductor. The critical current versus induction for the conductor is shown at $3.4 \mathrm{~K}, 4.2 \mathrm{~K}$, and $5.0 \mathrm{~K}$.)

The Lab G solenoid was operated at a current that was 11 percent over its design current at a current of $294 \mathrm{~A}$. The magnet quenched, and 2.96 MJ of stored magnetic energy was released into the magnet coils and the aluminum bobbin structure. At the time, there were only a few liters of liquid helium in the magnet's 300-liter helium vessel. As a result, the estimated temperature of the coil hot spot was about $4.8 \mathrm{~K}$. At the time of the quench, the magnet was being charged at the full voltage available from the power supply. Had the magnet been covered with liquid helium, it is probable that the magnet would have reached 300 A limit of the power supply.

It is clear that the focusing coils for MICE can be built using the same techniques used to build the Lab $\mathrm{G}$ coil. The inner radius of the coil would have to be increased to between 260 and $265 \mathrm{~mm}$, and the thickness of the aluminum bobbin inside the coil would have to be increased to at least $10 \mathrm{~mm}$. If the wet lay up winding technique is used for the MICE focusing coils, the radius of the coil current center would be about $300 \mathrm{~mm}$, which is less than the average current radius of $305 \mathrm{~mm}$ for the design shown in FIGURE 2. The thinner wet wound coil coupled with a thicker bobbin under the coils will produce lower stresses in the end plates of the magnet mandrel. It is clear that the focus coils can be fabricated as shown in FIGURE 2 or as shown in FIGURE 3. When the final design fabrication decisions are made, cost of the finished magnet will probably be a deciding factor.

The Lab G solenoid was designed to be a liquid bath cooled magnet. As a result, the helium reservoir volume was set at 300 liters. If this magnet were to be built again, one would not fabricate it as a bath cooled magnet, because the Fermilab safety committee's interpretation of the pressure vessel code makes the magnet too expensive to build. It cost more money to install the magnet in Lab $\mathrm{G}$ at Fermilab than it did to fabricate the magnet. Part of this cost was convincing the safety committee at Fermilab that the magnet was designed and tested in accordance with the pressure vessel code. The MICE focusing magnets will be cooled using a $4.4 \mathrm{~K}$ refrigerator. Cooling the magnet using forced two-phase helium in tubes attached to the coil package makes sense as long as there is a refrigerator and control cryostat (with its heat exchanger) available to do the cooling [8]. The problem of using a pure tubular cooling system for the focusing magnet system is the initial $4.2 \mathrm{~K}$ testing of the magnet in a location that does not have a helium refrigeration system. For this reason one might want to consider a hybrid approach that combines tubular cooling with a small helium bath (say 20 or 30 liters) to allow magnet testing at the place where the magnet is fabricated. 


\section{FORCES ON THE MICE FOCUSING MAGNETS}

Calculations of the magnetic forces on the focus coils show that forces on the focus coils are proportional to the average momentum of the muons in the channel squared. The largest force pushing the coil pair apart in the focusing module occurs in the center-focusing module of the MICE channel. This force is also proportional to the muon average momentum squared. During normal operation of the MICE channel there is no net cold to warm magnetic force on the center-focusing module. The end-focusing modules have a cold to warm force that must be carried by the magnet cold-mass support system. This force is induced by the field generated by the coupling coil on one side and the field generated by the detector module on the other side. This force is also proportional to the average muon momentum squared. There is a net force carried by the detector module magnet cold-mass support that is nearly the same as the net force carried by the end focus magnet cold-mass support system. Table 2 presents the results of magnetic force calculations on the MICE channel focusing magnets under various operating conditions for the channel.

TABLE 2. The calculated inter-coil magnetic forces and cold mass support system magnetic forces on the focusing magnet coils during various operating conditions for the MICE channel. The forces are calculated for average $\mu$ momenta of $200 \mathrm{MeV} / \mathrm{c}$ and $240 \mathrm{MeV} / \mathrm{c}$ for both the center and end focus modules.

Operating Case for MICE

Center Module, $200 \mathrm{MeV} / \mathrm{c}$

Center Module, $240 \mathrm{MeV} / \mathrm{c}$

End Module, $200 \mathrm{MeV} / \mathrm{c}$

End Module, $240 \mathrm{MeV} / \mathrm{c}, \mathrm{B}_{\mathrm{D}}=4 \mathrm{~T}$

End Module, $240 \mathrm{MeV} / \mathrm{c}, \mathrm{B}_{\mathrm{D}}=4.8 \mathrm{~T}$

Center, Coupling Quench, $200 \mathrm{MeV} / \mathrm{c}$

Center, Coupling Quench, $240 \mathrm{MeV} / \mathrm{c}$

End, Coupling Quench, $200 \mathrm{MeV} / \mathrm{c}$

End, Coupling Quench, $240 \mathrm{MeV} / \mathrm{c}, \mathrm{B}_{\mathrm{D}}=4 \mathrm{~T}$

End, Coupling Quench, $240 \mathrm{MeV} / \mathrm{c}, \mathrm{B}_{\mathrm{D}}=4.8 \mathrm{~T}$

\section{Inter-coil Force}

$(\mathrm{MN})^{*}$

1.822

2.624

1.784

2.569

2.569

1.335

1.922

1.441

2.075

2.075
Cold Mass Support

Force $(\mathrm{MN})^{*}$

0.000
0.000
0.184
$\sim 0.19$
0.265
0.000
0.000
0.393
$\sim 0.40$
0.566

* All forces given in this table are in the longitudinal direction (along the magnet axis). The sign of the force depends on the location of the focusing magnet module.

TABLE 2 shows forces from a coupling-coil quench when both coupling coils are in series [9]. A quench of the focus coils produces no net forces that are higher than the coil forces seen on the coils in the module before the quench. A quench of the detector module increases the inter-coil forces in the end modules to a value that is no higher than that of the center module. A quench in the detector module will reduce the cold mass support forces on the end focus modules. A quench of one detector module will put a small net force on the center module cold mass supports. Cases were shown with two values of the detector uniform field $B_{D} \cdot B_{D}=4 T$ assumes that the detector field does not change with average muon momentum. $\mathrm{B}_{\mathrm{D}}=4.8 \mathrm{~T}$ assumes that the detector field does change with muon average momentum. From the force calculations, it is clear that the cold mass support system for the focusing magnet must be designed for a longitudinal force of $0.6 \mathrm{MN}$ (about 60 metric tons).

Studies of the effects of coil placement errors on the forces seen by the focusing coils showed the following results: 1) An off axis displacement of one coil by $1.0-\mathrm{mm}$ produces a force of $3.3 \mathrm{kN}$ in the direction of the coil displacement and a torque of $0.33 \mathrm{kN} \mathrm{m}$. 2) An axial motion of one coil 1.0-mm changes the inter-coil force between coils about 0.5 percent. When the coils are moved apart, the force goes down. 3) A rotation of one coil 1-degree produces a side force of $5.8 \mathrm{kN}$ and a torque $2.7 \mathrm{kN} \mathrm{m}$. If the focus coil pair is manufactured to reasonable tolerances, the net misalignment forces will be small $(<300 \mathrm{~N})$. 4) If the coil pair is moved axially $1-\mathrm{mm}$, the net force in the axial direction is about $1.03 \mathrm{kN}$. 5) If the coil pair is moved radially $1-\mathrm{mm}$, the net force in the radial direction is about $-0.51 \mathrm{kN}$. If the coil pair is rotated 1-degree, the net torque in the direction of rotation is about $1.02 \mathrm{kN} \mathrm{m}$. 


\section{CONCLUSIONS}

The MICE focusing magnet is very much like the two-coil solenoid that was fabricated for the $800 \mathrm{MHz}$ RF cavity experiment in Lab G at Fermilab. With minor modifications, the Lab $\mathrm{G}$ solenoid fabrication method can be applied to the MICE focusing magnet. There appear to be at least two viable methods for manufacturing the MICE focusing solenoid magnets. The manufacturing method selected will depend on the fabrication cost and the cryogenic safety requirements of the Rutherford Appleton Laboratory (RAL).

The MICE focusing magnets can either be cooled in a helium bath with liquid helium supplied by a refrigerator or they can be cooled using force two-phase helium flowing in tubes on the outside of the coil package. The selection of the cooling method depends on cost of magnet fabrication, the cost of testing the magnet at the fabrication site, and costs imposed by RAL safety standards.

It is recommended that the design inter-coil force for the MICE coil pair be at least $3 \mathrm{MN}$ (300 metric tons). This is the projected inter-coil force if the magnet is operated in the gradient mode at its short sample current at $4.2 \mathrm{~K}$. The design force for the cold mass support system for the focusing magnet module should be greater than 0.6 MN (60 metric tons). If the MICE focusing magnet module is manufactured to reasonable tolerances, the forces produced by misalignments will be small compared to the forces that the magnet system must be designed for.

\section{ACKNOWLEDGEMENTS}

This work was performed at the Lawrence Berkeley National Laboratory with the support of the Office Science, United States Department of Energy under DOE contract DEAC03-76SF00098.

\section{REFERENCES}

1. R. B. Palmer and R. Fernow, "200 MHz Cooling Experiment Design Version 4," the 23 October 2002 version of the MICE channel.

2. M. A. Green and J. M. Rey, "Superconducting Solenoids for an International Muon Cooling Experiment," IEEE Transactions on Applied Superconductivity 13, p 1373, (2003)

3. M. A. Green, E. L. Black, M. A. Cummings, et al., "The Integration of Liquid and Solid Muon Absorbers into a Focusing Magnet of a Muon Cooling Channel," Proceedings of the 3003 Particle Accelerator Conference, Portland OR, IEEE Publications (2003)

4. M. A. Green, "The Superconducting Solenoid Magnets for MICE," Lawrence Berkeley Laboratory report LBNL-51920, December (2002)

5. M. A. Green, S. Ishimoto, W. Lau and S. Yang, "A Heat Exchanger between Forced Flow Helium Gas at 14 to $18 \mathrm{~K}$ and Liquid Hydrogen at $20 \mathrm{~K}$ circulated by Natural Convection," Advances in Cryogenic Engineering 49, (This Publication), AIP Press, New York (2003)

6. M. A. Green, J. Y. Chen and S. T. Wang, "The Design and Fabrication of a Gradient Solenoid for the High Powered RF Cavity Experiment for the Muon Collider," Institute of Physics Conference Series, Number 167, Institute of Physics Publishing LTD, Bristol, (2000)

7. M. A. Green, J. Y. Chen and S. T. Wang, "A Test of a Superconducting Solenoid for the Mucool RF Experiment," IEEE Transactions on Applied Superconductivity 11, No. 1, p 2296, (2001)

8. M. A. Green, et al., "Operation of a Forced Two-phase Cooling System on a Large Superconducting Magnet," Proceedings of the International Cryogenic Engineering Conference 8, Genoa Italy, p 72, Butterworth Press, Oxford UK (1980)

9. J. H. Rochford and E Baynham, "Forces Induced by Coil Misalignments in MICE," a preliminary report form the Rutherford Appleton Laboratory, June 2003 
This document was prepared as an account of work sponsored by the United States Government.

While this document is believed to contain correct information, neither the United States Government nor any agency thereof, nor The Regents of the University of California, nor any of their employees, makes any warranty, express or implied, or assumes any legal responsibility for the accuracy, completeness, or usefulness of any information, apparatus, product, or process disclosed, or represents that its use would not infringe privately owned rights. Reference herein to any specific commercial product, process, or service by its trade name, trademark, manufacturer, or otherwise, does not necessarily constitute or imply its endorsement, recommendation, or favoring by the United States Government or any agency thereof, or The Regents of the University of California. The views and opinions of authors expressed herein do not necessarily state or reflect those of the United States Government or any agency thereof, or The Regents of the University of California.

Ernest Orlando Lawrence Berkeley National Laboratory is an equal opportunity employer. 